\title{
Impact of elevated core temperature on cognition in hot environments within a military context
}

\author{
Edward Tom Ashworth ${ }^{1}$ (i) $\cdot$ James David Cotter ${ }^{2} \cdot$ Andrew Edward Kilding $^{1}$
}

Received: 23 June 2020 / Accepted: 17 December 2020 / Published online: 11 January 2021

(C) The Author(s), under exclusive licence to Springer-Verlag GmbH, DE part of Springer Nature 2021

\begin{abstract}
Purpose Cognition can be impaired during exercise in the heat, potentially contributing to military casualties. To our knowledge, the independent role of elevated core temperature during exercise has not been determined. The aim of the current study was to evaluate effects of elevated core temperature on cognition during physically encumbering, heated exercise, and to determine whether the perceptual cooling effects of menthol preserves cognition.

Methods Eight participants complete three trials in randomised order: one normothermic (CON) and two with elevated $\left(38.5^{\circ} \mathrm{C}\right)$ core temperature, induced by prior immersion in neutral versus hot water The CON trial and one hot trial (HOT) used a water mouth-rinse following each cognitive task of the trial, (HOT) while the other used a menthol mouth-rinse (MENT). Participants walked in humid heat $\left(33^{\circ} \mathrm{C}, 75 \%\right.$ relative humidity) in military clothing, completing a cognitive battery of reaction time, perceptual processing, working memory, executive function, cognitive flexibility, vigilance, and declarative memory.

Results No differences in cognitive performance were observed between any conditions. Near-infrared spectroscopy showed greater oxygenated haemoglobin tissue content in HOT and MENT compared to CON $\left(\Delta \mathrm{O}_{2} \mathrm{Hb}_{-}-\mathrm{deO}_{2} \mathrm{Hb}: 2.3 \pm 4.5 \mu \mathrm{M}\right.$, $p<.024)$, and lower deoxygenated haemoglobin in MENT than in CON or HOT $(p=.017)$, suggesting higher brain metabolism during the more stressful conditions.

Conclusion Moderately elevated core $\left(38.5^{\circ} \mathrm{C}\right)$ and skin temperature does not appear to impair cognitive performance during exercise despite mildly elevated cerebral metabolism. The effects of menthol remain undetermined due to the lack of heat-mediated cognitive impairment.
\end{abstract}

Keywords Cognitive $\cdot$ Physiology $\cdot$ Menthol $\cdot$ Exercise

$\begin{array}{ll}\text { Abbreviations } \\ \text { CON } & \text { Control group } \\ \text { HOT } & \text { Hot group } \\ \text { MENT } & \text { Menthol group } \\ \text { WI } & \text { Water immersion (figure only) } \\ \text { W } & \text { Water mouth-rinse (figure only) }\end{array}$

Communicated by George Havenith.

Edward Tom Ashworth

Edward.Ashworth@aut.ac.nz

1 Sports Performance Research Institute New Zealand (SPRINZ), Auckland University of Technology, 17 Antares Place, Rosedale, Auckland 0632, New Zealand

2 School of Physical Education, Sport and Exercise Sciences, University of Otago, 55/47 Union Street W, North Dunedin, Dunedin 9016, New Zealand $\begin{array}{ll}\text { M } & \text { Menthol mouth-rinse (figure only) } \\ \text { TLX } & \text { Task-load index }\end{array}$

\section{Introduction}

Military operations require not only physical performance (Birrell and Haslam 2010, Hunt et al. 2016), but also cognitive performance (Vrijkotte et al. 2016, Martin et al. 2019, Martin et al. 2020). Cognitive impairment accounts for most accidents in both training and battle (Vrijkotte et al. 2016), and declines faster than physical function during sustained operations (Lieberman et al. 2006). Extreme environments further restrict availability of cognitive resources (Vasmatzidis et al. 2002, Hancock and Vasmatzidis 2003).

While military operations take place in various climates, heat is increasingly prevalent and concerning. Poor decision making is more frequent in hot environments (Froom et al. 
1993, Martin et al. 2019), and although has been assumed to be as a function of elevated core temperature (Hocking et al. 2001, Schmit et al. 2017, Martin et al. 2019), it could also be related to perceptual and/or cardiovascular effects of high skin and mean body temperature (Gaoua 2010, Gaoua et al. 2017), dehydration (Cian et al. 2001, Ganio et al. 2011, Nolte et al. 2013, Cvirn et al. 2019), nutritional status (van Dokkum et al. 1996, Owen et al. 2004, Bandelow, Maughan et al. 2010), experience (Hancock and Vasmatzidis 2003), solar radiation (Piil et al. 2020), physical (Grego et al. 2005, Eddy et al. 2015, Yanovich et al. 2015, Bhattacharyya et al. 2017) and cognitive fatigue (Lieberman et al. 2006, Head et al. 2017), carried loads (Williams et al. 1997) and sleep deprivation (Lieberman et al. 2005), among other factors. The role of core temperature on cognition remains unclear. Some researchers suggest that high $\left(>38.5^{\circ} \mathrm{C}\right)$ core temperature impairs complex cognition (Gaoua et al. 2011, Piil et al. 2017, Schmit et al. 2017), whereas others suggest the direction of change in core temperature has more influence on cognition (Allan and Gibson 1979, Allan et al. 1979, Hancock and Vasmatzidis 2003). Furthermore, brain activity has been shown to alter during passive hyperthermia (Jiang et al. 2013, Liu et al. 2013, Qian et al. 2013, Xue et al. 2018), suggesting an inherent ability to mitigate effects of high temperature and potentially preserve performance. Therefore, the aim of this study was to isolate the effects of elevated core temperature within exercise and determine its influence on cognitive performance within a military context.

If core temperature plays a major role in impairing cognition, then the effects of heat could be minimised by cooling garments or heat acclimation strategies (Patterson et al. 1998, Hemmatjo et al. 2017). For example, head cooling and cooling collars have been successful in overcoming the negative effects of heat on cognition (Gaoua et al. 2011, Lee et al. 2014). Recently, the use of a menthol mouth-rinse, which provides perceptual cooling, has been shown to improve physical performance (Mündel and Jones 2010, Stevens et al. 2016, Flood et al. 2017). It is also more operationally feasible than most cooling methods. Therefore, a secondary aim was to determine whether menthol mouth-rinse could preserve cognitive function within a military context in the heat.

\section{Methods}

A randomised, repeated measures, cross-over design was employed, during which each participant completed three experimental sessions assessing cognitive function during military-specific exercise in the heat (Fig. 1), after familiarisation with the cognitive battery. The sessions were comprised of a normothermic session (CON) and two hyperthermic sessions. One of the hyperthermic sessions included repeated mouth-rinse using a menthol solution (MENT), which was controlled for in the other hyperthermic trial using an identical volume water mouth-rinse (HOT), which also occurred in CON. During each session, cognitive, physiological, and perceptual and cognitive measures were recorded. Each experimental session was completed at the same time of day for each participant, with at least 1 week, but no more than 3 weeks, between each trial. With institutional ethical approval (AUTEC 19/368), and in accordance with the Declaration of Helsinki, participants provided written, informed consent.

\section{Participants}

Eight participants (three males, five females) took part in the study. A sample of 16 was initially desired to provide a statistical power of 0.8 for detecting a medium effect size with an alpha value of 0.05. Due to COVID-19 restrictions, only eight participants completed the study, providing a statistical power of 0.39 for medium effect sizes, and 0.79 for large effect sizes. Participants were habitually active, averaging $289 \pm 148 \mathrm{~min}$ of structured exercise per week. Female participants completed each condition during the follicular phase of the menstrual cycle, as confirmed by selfreported menstruation. No female participants were using oral contraceptives.

\section{Familiarisation}

Prior to any experimental session, participants were required to undergo a familiarisation session lasting $30 \mathrm{~min}$. During this time, the tasks were explained in full, and participants practiced all cognitive tasks until both the participant and experimenters were satisfied that any further learning effects were minimal.

\section{Experimental session}

\section{Pre-heating}

Upon arrival in the laboratory participants provided a urine sample and privately inserted a rectal thermometer (Hinco Instruments, Australia) $12 \mathrm{~cm}$ beyond the anal sphincter. Participants were then immersed in $40{ }^{\circ} \mathrm{C}$ water until their rectal temperature reached $38.5^{\circ} \mathrm{C}$ (in HOT and MENT), or in $36^{\circ} \mathrm{C}$ in $\mathrm{CON}$ for a similar length of time. At the onset of heating, participants completed a simple reaction time task (Novak 2016), requiring a response when a red circle appeared but avoiding responding to blue and black circles. The test was used to provide a baseline of cognition to be compared between sessions. During the pre-heating, perceptual measures of thermal discomfort (1-10) (Bedford 1936), thermal sensation (1-13) (ISO 1994), feeling $(-5-+5)$ 
Fig. 1 Schematic representation of the experimental design. In each experimental condition participants were initially immersed in water (WI), either heated to $40^{\circ} \mathrm{C}$ or $36^{\circ} \mathrm{C}$. Following this they walked in the heat while completing a cognitive testing battery. Following each task participants were prescribed a mouth-rinse of either water (W) or menthol (M) during a $\sim 1$ min interlude

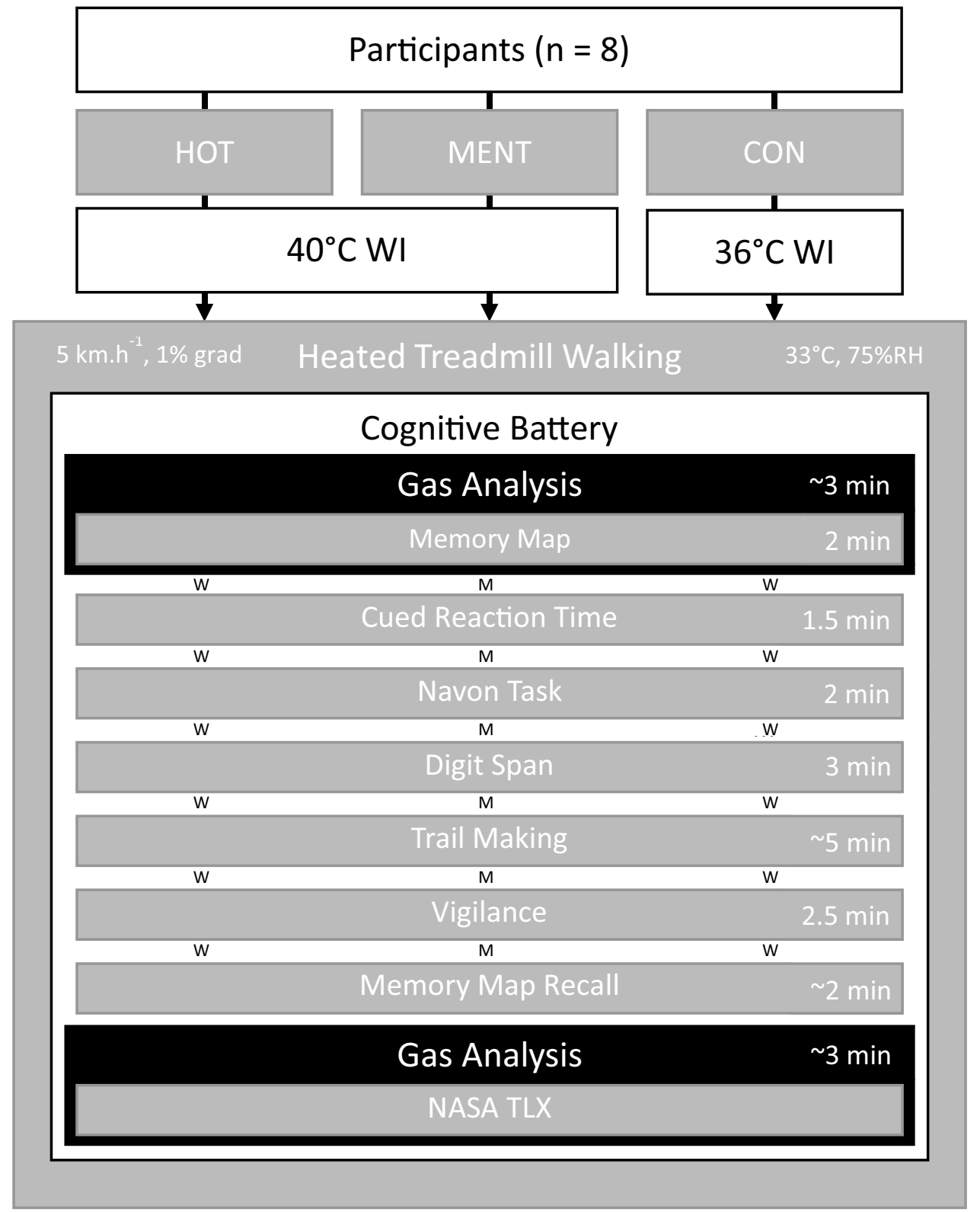

(Hardy and Rejeski 1989) and sleepiness (1-9) (Hoddes 1972) were recorded alongside heart rate at $37.5^{\circ} \mathrm{C}, 38.0^{\circ} \mathrm{C}$ and $38.5^{\circ} \mathrm{C}$, or at corresponding times during the control phase. The length of the CON session was determined by the time each individuals rectal temperature took to reach $38.5^{\circ} \mathrm{C}$ in preceding trials, or in the event of the CON session occurring first, based on a rate of increase of $0.4^{\circ} \mathrm{C}$ per 10 min established a-priori during pilot trials.

\section{Transition to exercise in heat}

When rectal temperature reached $38.5^{\circ} \mathrm{C}$, or similar time had passed in $\mathrm{CON}$, participants exited the water and immediately dried off. This $38.5^{\circ} \mathrm{C}$ temperature was used as the onset for walking because it has been shown to impair cognitive function (Schmit et al. 2017), and it allowed core temperature to continue rising but not exceed the ethical limit of $39.5^{\circ} \mathrm{C}$ before completing the protocol. Skin temperature sensors were then attached to the participant's chest, bicep, thigh, and calf before they donned military clothing (long-sleeved shirt and trousers) and their own shoes. Participants were then escorted to the laboratory and weighed before entering the heat chamber (Design Environmental, Simultech Australia, Australia) set to $33^{\circ} \mathrm{C}$ and $75 \%$ relative humidity. A weighted body armour vest (20 kg) was worn over the military clothing and a near-infrared spectroscopy (NIRS) sensor was placed onto the forehead (further information below). Participants were then instructed to walk 
on a motorised treadmill (Platinum Club Series, Life Fitness, Illinois, USA) for $30 \mathrm{~min}$ at $5 \mathrm{~km} \cdot \mathrm{h}^{-1}$ and $1 \%$ gradient. Expired gas was collected during the first $4 \mathrm{~min}$ and final 3 min of the walk using a calibrated metabolic system (TrueOne 2400, Parvo Medics, Utah, USA).

\section{Menthol supplementation}

Prior to each task participants were given a $25 \mathrm{~mL}$ mouthrinse with either water (in CON and HOT) or $0.1 \%$ concentration (Best et al. 2018) menthol (Pure Nature, Auckland, NZ) in MENT. Total fluid volume was $150 \mathrm{~mL}$ in each condition (Fig. 1). Participants were instructed to swill the solution for $5 \mathrm{~s}$, before spitting it into a bowl. The menthol solution was prepared by mixing menthol
Fig. 2 Physiological measures during cognitive testing while walking in military dress in the heat $\left(33^{\circ} \mathrm{C}, 75 \% \mathrm{RH}\right)$ while either normothermic (CON), hyperthermic (HOT) or hyperthermic with the aid of a menthol mouth-rinse (MENT). Measures of rectal temperature (a), skin temperature (b), and heart rate (c) were all obtained throughout the trial. Data are reported as mean $\pm \mathrm{SD}$. In a thick lines represent means, while thin lines display individual responses. In $\mathbf{b}$ and $\mathbf{c}$ MENT and HOT results are offset along the time axis for clarity. *Indicates $p<.05$ between CON and HOT. "Indicates $p<.05$ between CON and MENT
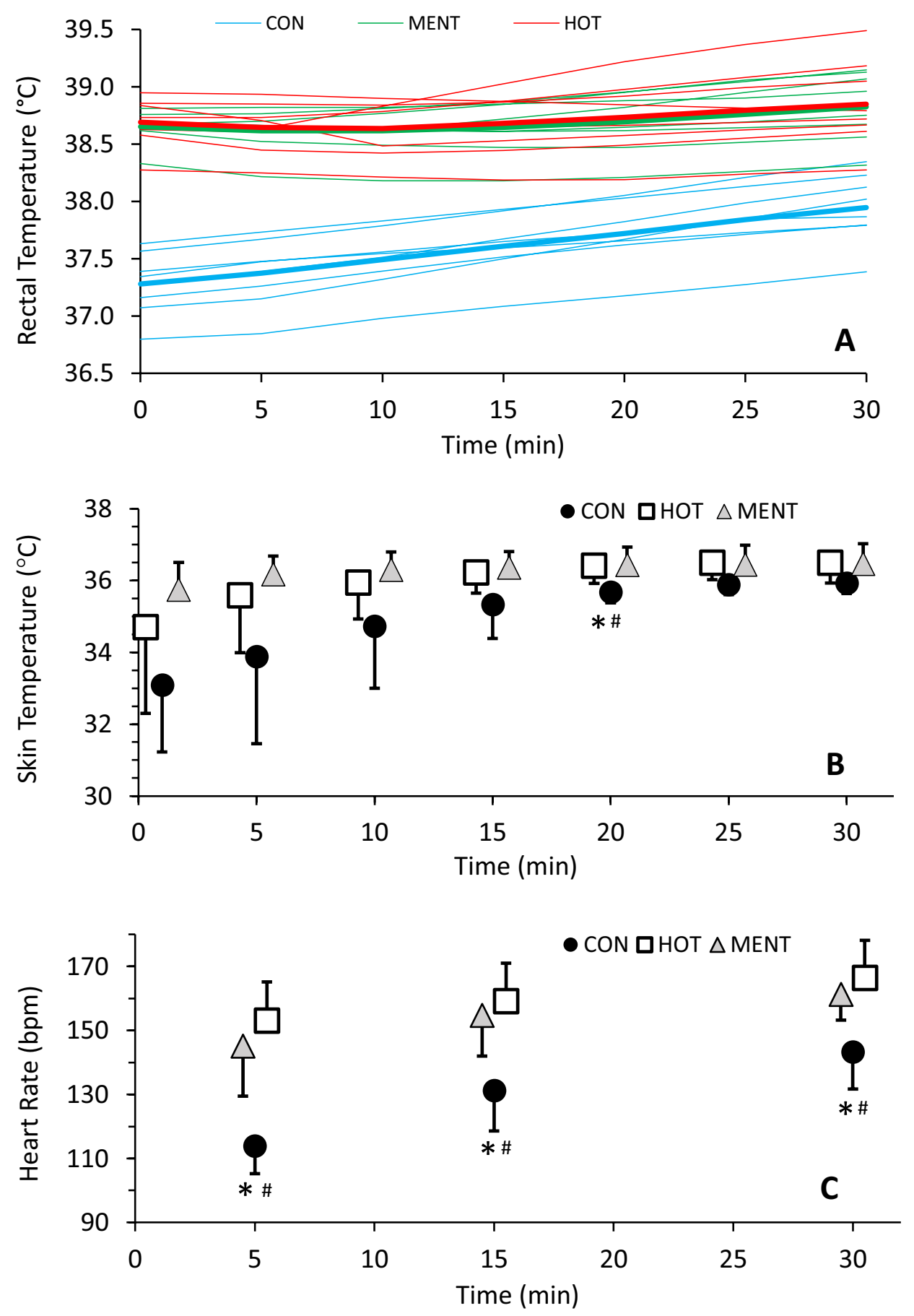
with distilled water at a $1: 999$ ratio, heating it to $50{ }^{\circ} \mathrm{C}$ and stirring for $2 \mathrm{~h}$ before storing at room temperature (Fig. 2).

\section{Cognitive testing}

Cognitive tasks were randomised for each participant using either in-built randomisation for computer tasks, or Latin squares for paper-based tasks.

\section{Declarative memory}

The memory task involved participants being asked to memorise a fictional map of an urban environment. The map detailed a prescribed route through several streets to reach a target house. The list of questions that would later be asked was provided alongside the map to minimise learning effects. Participants were given 2 min to study the map before it was removed. Then, 25 min later, the questions were read aloud, and participants asked to correctly answer as many as possible, up to a maximal score of 22 .

\section{Reaction time (go/no-go)}

A cued reaction time task was presented to assess go/no-go reaction time (Posner and Cohen 1984). A box was presented on each side of the screen with a focal point in the middle. Participants were asked to focus on the focal point and respond to a 'GO' signal presented in one of the boxes, by tapping the left, or right, key. Immediately prior to the 'GO' signal appearing a cross appeared in one of the boxes, cueing the response. The cue was in the same box as the 'GO' signal $75 \%$ of the time. Forty trials were run, allowing calculation of reaction times for both cued and uncued stimuli, as well as accuracy rates.

\section{Perceptual processing}

The Navon task was used to evaluate perceptual processing (Navon 1977). Large letters, constructed from repeated smaller letters, were presented one at a time on the screen in front of participants. Participants then had to determine whether the letter ' $\mathrm{H}$ ' or ' $\mathrm{O}$ ' was present in the image by responding yes or no on a keyboard. Forty images were presented, from which reaction times and correct response rates were calculated when the stimuli were global, local, and when none was present.

\section{Working memory}

Digit span was used to assess working memory (Conklin et al. 2018). A series of numbers was read out and participants asked to recall them in the reverse order to that in which they were read (i.e. 123 becomes 321). Spans started at three digits long and progressed after a correct response. After three incorrect responses at a single level the test was stopped. The last successful span was recorded.

\section{Executive function and cognitive flexibility}

A trail making task was used to assess executive function (Arbuthnott and Frank 2000). For task A, participants were given a sheet with numbers 1-25 inside their own circles randomly scattered across the page. The numbers then had to be connected using a pencil, in order and as quickly as possible without passing through other circles. Following this task, participants were presented a similar task (task B) with numbers 1-13 and letters A-L. In this task, participants had to switch sets, alternating between numerical and alphabetical (i.e. 1A2B3C). For both tasks, time to completion was recorded, as well as errors of the numbering order, and errors going through other circles.

\section{Vigilance}

The Mackworth clock task was used to assess vigilance (Mackworth 1948, Giambra et al. 2013). Participants monitored a clock hand ticking around the screen. When the clock hand skipped a second participants responded by pressing a button on the keyboard. Correct and incorrect reaction times, as well as percentage of correct responses were recorded.

\section{NASA task-load index}

Following the recall of the memory items, participants were connected to the gas analysis for a further 3 min. During this time, they were presented with a NASA task-load index (TLX) to complete, giving information on the mental, temporal, physical demands and well as performance, effort and frustration.

\section{Physiological and perceptual measures}

Heart rate (Platinum Club Series, Life Fitness, Illinois, USA) and perceptual measures of thermal discomfort, thermal sensation, feeling, sleepiness and rating of perceived exertion (RPE: 15-point scale ranging 6-20) (Borg 1982) were recorded at 5, 15 and $30 \mathrm{~min}$ into the walk. Following the walk, a second urine sample was taken. Samples were analysed for urine specific gravity using a urine refractometer (Atago, Japan) to provide hydration status.

Rectal and skin temperature were recorded at $1 \mathrm{~Hz}$ (SQ2020, Grant Instruments, Cambridge, UK). In preparation for analysis, rectal and skin temperature readings were filtered due to noise caused by connections with the logger 
and occasional skin temperature probes losing contact with the skin due to the humid microenvironment. A filter was applied removing all readings that changed by more than $0.1^{\circ} \mathrm{C} . \mathrm{s}^{-1}$. Then, a low-pass Butterworth filter of $0.02 \mathrm{~Hz}$ was applied to the data. Missing data were filled with linear interpolation. Mean skin temperature was calculated using the following formula (Ramanathan 1964):

$\mathrm{T}_{\text {Sk }}=0.3 \mathrm{~T}_{\text {Chest }}+0.3 \mathrm{~T}_{\text {Bicep }}+0.2 \mathrm{~T}_{\text {Thigh }}+0.2 \mathrm{~T}_{\text {Calf }}$

If a thermistor became askew, the equation was modified to compensate the weights of the three remaining sensors proportionally to maintain the summation of coefficients to 1.0 (i.e. $\mathrm{T}_{\mathrm{Sk}}=0.375 \mathrm{~T}_{\text {Chest }}+0.375 \mathrm{~T}_{\text {Bicep }}+0.25 \mathrm{~T}_{\text {Thigh }}$ if the calf reading was lost). If two sensors produced no signal, no temperature was calculated. Averages over 5-min periods were used for analysis, with the relative difference between each analysis point and the prior analysis point used to determine the direction and magnitude of change in rectal temperature.

\section{Near-infrared spectroscopy analysis}

NIRS was used as a practical method of collecting cerebral adjustments within an ambulatory setting (Boone et al. 2015). NIRS allows measurement of oxygenated and deoxygenated haemoglobin within the cerebral tissue of the prefrontal cortex (Suzuki et al. 2004, Strangman et al. 2006). A NIRS sensor was placed $\sim 1 \mathrm{~cm}$ above the eyebrow and held in place by an opaque headband that prevented light interference throughout the walk in the heat chamber. Data were logged by an oximeter at $2 \mathrm{~Hz}$ (OxiplexTS, ISS, Champaign, IL). A Butterworth filter of $0.01 \mathrm{~Hz}$ was applied to all data to remove noise, mostly from movement artefact. Data were then standardised to the initial $5 \mathrm{~min}$ of cognitive testing to provide a baseline, allowing subsequent calculation of baseline-adjusted data points every $5 \mathrm{~min}$, using the accumulative average of the prior $5 \mathrm{~min}$. The estimated oxygenation difference was calculated as the baseline-adjusted difference between oxygenated haemoglobin and deoxygenated haemoglobin.

\section{Statistical analysis}

All analyses were conducted in $\mathrm{R}$ version 3.6.1 ( $\mathrm{R}$ foundation for Statistical Computing, Vienna, Austria). For all variables, a mixed model was used from the lmer package (Bates et al. 2014), with session (chronological session number) and condition as fixed effects, with participant as the random effect. Planned pairwise comparisons were then carried out on the mixed model using the emmeans package with condition as the defining variable. All values are reported as the model's estimated mean \pm standard deviation.
It has been suggested that the direction of core temperature movement has a greater role than the absolute core temperature during cognitive tasks (Allan and Gibson 1979, Allan et al. 1979). Therefore, posteriori analyses were carried out to investigate the relationship between the direction of change in rectal temperature and cognitive performance. This involved re-running the analysis, for only the observations when rectal temperature was higher than it had been at the previous time point. A second posteriori analysis used the same method but only if the rate of rise in rectal temperature was $>0.5^{\circ} \mathrm{C} \mathrm{h}^{-1}$.

\section{Results}

\section{Pre-heating}

Duration immersed at the start of each session averaged $36.6 \pm 5.7 \mathrm{~min}$ (range 29.5-41 $\mathrm{min}$ ) and was not different between conditions (all $p>0.523$ ). Core temperature was not different at baseline between conditions (all $p>0.481$ ) and increased in HOT and MENT (both $p<0.001$ ) but not in CON $(p=0.911)$. Perceptions of thermal sensation, thermal discomfort and feeling deteriorated in HOT and MENT compared to CON (all $p<0.001$ ). There were no differences between MENT and HOT (all $p>0.264$ ) other than sleepiness being 1.0 $\pm 0.8 \mathrm{AU}$ higher in MENT $(p=0.002)$, which was also elevated $1.3 \pm 0.8 \mathrm{AU}$ compared to CON $(p<0.001)$. Heart rate was $31 \pm 13 \mathrm{bpm}$ higher in HOT and MENT $(p<0.001)$ than CON, with no differences between HOT and MENT $(p=0.776)$. Fluid consumption was $0.3 \pm 0.1 \mathrm{~L}$ higher in HOT and MENT $(0.5 \pm 0.3 \mathrm{~L})$ than in CON (CON: $0.2 \pm 0.3 \mathrm{~L}$, both $p<0.001$ ) but this did not affect hydration status (all $p>0.552$ ). Baseline cognition was similar between conditions (all $p>0.827$ ).

\section{Physiological responses during exercise in the heat}

In relation to cognitive task performance, rectal temperature was increasing in $50 \%$ of trials during the memory task presentation (12/24), 63\% during the cued reaction time task (15/24), $79 \%$ during the Navon task (19/24), and $>95 \%$ in the tasks that followed ( $>23 / 24)$. The slope of rectal temperature was $\sim 1^{\circ} \mathrm{C} . \mathrm{h}^{-1}$ steeper during CON $\left(\mathrm{CON}: 1.3 \pm 0.4^{\circ} \mathrm{C}\right.$. $\mathrm{h}^{-1}$ ) compared to HOT or MENT (HOT: $0.3 \pm 0.7^{\circ} \mathrm{C} . \mathrm{h}^{-1}$; MENT: $0.3 \pm 0.4^{\circ} \mathrm{C} . \mathrm{h}^{-1}$; CON vs HOT: $p<0.001$; CON vs. MENT: $p<0.001$; MENT vs. HOT: $p=0.946$ ), but attained only $37.9^{\circ} \mathrm{C}$ by completion of exercise.

There were no differences between conditions in $\mathrm{VO}_{2}$ (CON: $16.3 \pm 1.1 \mathrm{~mL} \cdot \mathrm{kg}^{-1} \cdot \mathrm{min}^{-1}$; HOT: $16.2 \pm 1.4 \mathrm{~mL} \cdot \mathrm{kg}^{-1}$. $\mathrm{min}^{-1}$; MENT: $16.1 \pm 1.7 \mathrm{~mL} \cdot \mathrm{kg}^{-1} \cdot \mathrm{min}^{-1}$; all $p=1.000$ ), 
Table 1 Cognitive performance while walking on a treadmill whilst wearing military dress in the heat $\left(33^{\circ} \mathrm{C}, 75 \% \mathrm{RH}\right)$ while either normothermic (CON), hyperthermic (HOT) or hyperthermic with the aid of a menthol mouth-rinse (MENT)

\begin{tabular}{|c|c|c|c|c|c|c|c|}
\hline \multirow[t]{2}{*}{ Task } & \multicolumn{3}{|c|}{ Mean \pm standard deviation } & \multirow[t]{2}{*}{$p$ value } & \multicolumn{3}{|l|}{ Effect sizes } \\
\hline & $\mathrm{CON}$ & HOT & MENT & & CON vs. HOT & CON vs. MENT & HOT vs. MENT \\
\hline \multicolumn{8}{|l|}{ Reaction time (go/no-go) } \\
\hline Cued RT (ms) & $321 \pm 52$ & $311 \pm 32$ & $305 \pm 43$ & $>.486$ & 0.23 & 0.34 & 0.16 \\
\hline Uncued RT (ms) & $374 \pm 61$ & $361 \pm 51$ & $369 \pm 62$ & $>.999$ & 0.23 & 0.08 & 0.14 \\
\hline Correct $(\%)$ & $96 \pm 4$ & $95 \pm 6$ & $96 \pm 4$ & $>.999$ & 0.20 & 0.00 & 0.20 \\
\hline \multicolumn{8}{|l|}{ Perceptual processing } \\
\hline Global RT (ms) & $731 \pm 129$ & $688 \pm 116$ & $721 \pm 144$ & $>.482$ & 0.35 & 0.07 & 0.25 \\
\hline Local RT (ms) & $716 \pm 156$ & $714 \pm 171$ & $692 \pm 126$ & $>.999$ & 0.01 & 0.17 & 0.15 \\
\hline None RT (ms) & $802 \pm 224$ & $724 \pm 113$ & $778 \pm 186$ & $>.399$ & 0.44 & 0.12 & 0.35 \\
\hline Correct (\%) & $96 \pm 4$ & $92 \pm 5$ & $93 \pm 7$ & $>.716$ & 0.88 & 0.53 & 0.16 \\
\hline \multicolumn{8}{|l|}{ Working memory } \\
\hline Digit span 1st error (level) & $7.3 \pm 1.2$ & $6.9 \pm 1.2$ & $6.8 \pm 1.0$ & $>.999$ & 0.33 & 0.45 & 0.09 \\
\hline Maximum span (level) & $7.3 \pm 1.0$ & $6.8 \pm 0.7$ & $7.3 \pm 0.7$ & $>.316$ & 0.58 & 0.00 & 0.71 \\
\hline \multicolumn{8}{|l|}{ Executive function } \\
\hline Trail-making A (min) & $1.1 \pm 0.3$ & $1.1 \pm 0.5$ & $1.1 \pm 0.2$ & $>.999$ & 0.00 & 0.00 & 0.00 \\
\hline Trail-making B (min) & $1.2 \pm 0.5$ & $1.2 \pm 0.7$ & $1.2 \pm 0.4$ & $>.999$ & 0.00 & 0.00 & 0.00 \\
\hline Trail-making errors & $2.6 \pm 3.5$ & $5.4 \pm 7.3$ & $2.1 \pm 2.6$ & $>.445$ & 0.49 & 0.16 & 0.60 \\
\hline \multicolumn{8}{|l|}{ Vigilance } \\
\hline False starts & $1.5 \pm 1.2$ & $4.2 \pm 3.0$ & $2.6 \pm 2.6$ & $>.056$ & 1.18 & 0.54 & 0.57 \\
\hline Correct RT (ms) & $491 \pm 43$ & $521 \pm 59$ & $496 \pm 50$ & $>.672$ & 0.58 & 0.11 & 0.46 \\
\hline Correct (\%) & $79 \pm 8$ & $68 \pm 16$ & $78 \pm 14$ & $>.087$ & 0.87 & 0.09 & 0.67 \\
\hline \multicolumn{8}{|l|}{ Memory } \\
\hline Correct responses & $14.4 \pm 1.9$ & $8.3 \pm 4.9$ & $12.6 \pm 3.7$ & $>.116$ & 1.64 & 0.61 & 0.99 \\
\hline
\end{tabular}

$p$ values display the results when comparing between all conditions from pairwise comparisons

$\mathrm{VCO}_{2}\left(\mathrm{CON}: 1.01 \pm 0.16\right.$ L. $\mathrm{min}^{-1}$; HOT: $0.98 \pm 0.25$

L. $\mathrm{min}^{-1}$; MENT: $0.97 \pm 0.23 \mathrm{~L} \cdot \mathrm{min}^{-1}$; all $p>0.517$ ) and RER (CON: $0.88 \pm 0.05$; HOT: $0.85 \pm 0.06$; MENT: $0.85 \pm 0.07$; all $p>0.214)$.

\section{Perceptual responses}

Thermal discomfort, feeling, sleepiness and rating of perceived exertion all deteriorated in HOT and MENT compared to CON (all $p>0.001$ ), with no differences
Fig. 3 Perceptual responses to cognitive tasks during a 30-min military-dressed heated treadmill walk in humid heat $\left(33^{\circ} \mathrm{C}\right.$, $75 \% \mathrm{RH})$ while either normothermic $(\mathrm{CON})$, hyperthermic (HOT) or hyperthermic with the aid of a menthol mouthrinse (MENT). Perceptions consist of the NASA Task-Load Index (TLX) for cognitive tasks, as well as common heatrelated perceptions. Feeling is normalised to a positive scale. ${ }^{\alpha}$ Indicates $p<.05$ between CON and HOT, ${ }^{\beta}$ Indicates $p<.05$ between CON and MENT, no differences were observed between MENT and HOT

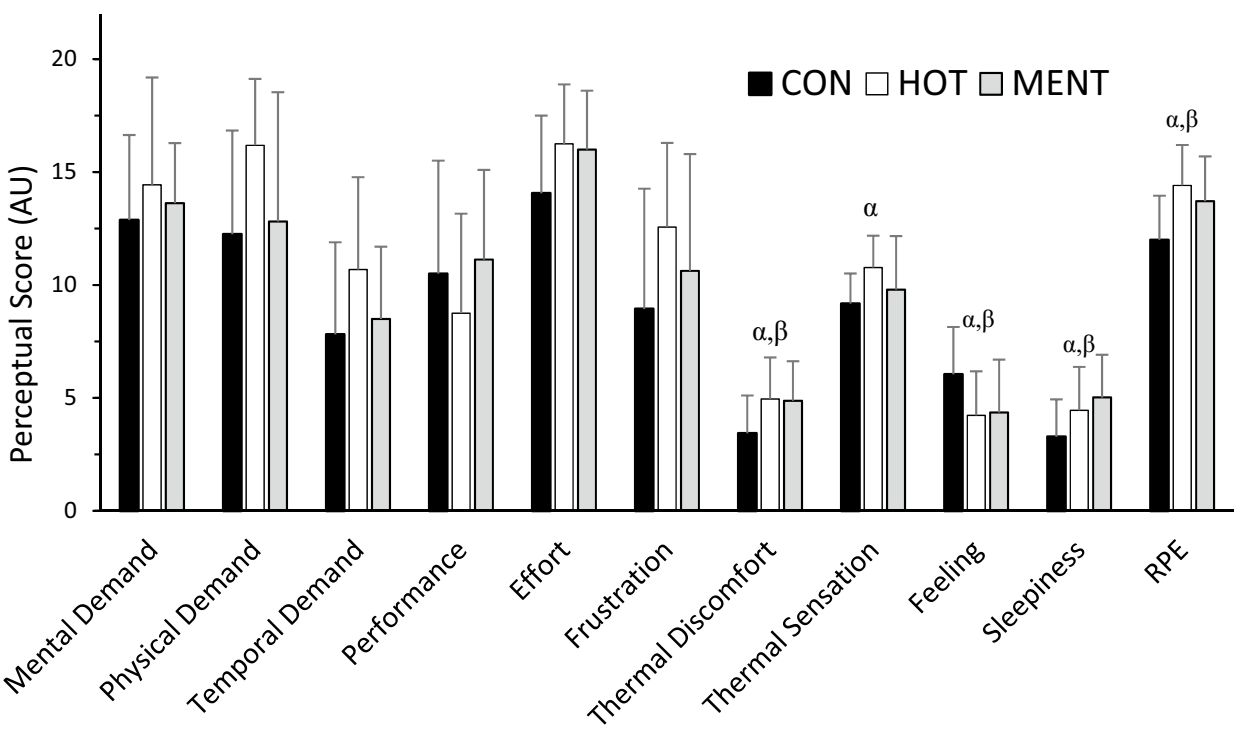


between MENT and HOT (all $p>0.174$ ) (Fig. 3). Thermal sensation was $2 \pm 1 \mathrm{AU}$ greater in the HOT than the CON condition (CON: $8.8 \pm 1.4$; HOT: $10.8 \pm 1.3$; MENT: $9.8 \pm 1.8$; CON vs. HOT: $p=0.001$; CON vs. MENT: $p=0.111$; MENT vs. HOT: $p=0.111$ ). No differences were seen between conditions in any aspect of the NASA task-load index (all $p>0.191$ ).

\section{Near-infrared spectroscopy}

There was a greater increase in oxygenated haemoglobin of $2.0 \pm 4.2 \mu \mathrm{M}$ in HOT and $2.5 \pm 4.3 \mu \mathrm{M}$ in MENT compared to the CON trial (CON: $0.3 \pm 3.7 \mu \mathrm{M}$; HOT: $2.3 \pm 4.8 \mu \mathrm{M}$; MENT: $2.8 \pm 5.0 \mu \mathrm{M}$; CON vs. HOT: $p=0.024$; CON vs. MENT: $p=0.009$; HOT vs. MENT: $p=0.510$ ). Deoxygenated haemoglobin was $0.7 \pm 1.1 \mu \mathrm{M}$ lower in the MENT trial than CON and $0.6 \pm 1.3$ lower than HOT (CON: $0.4 \pm 1.2 \mu \mathrm{M}$; HOT: $0.3 \pm 1.5 \mu \mathrm{M}$; MENT: $-0.3 \pm 1.1 \mu \mathrm{M}$; CON vs. HOT: $p=0.823$; CON vs. MENT: $p=0.017$, HOT vs. MENT: $p=0.017)$. However, estimated oxygenation difference (oxygenated
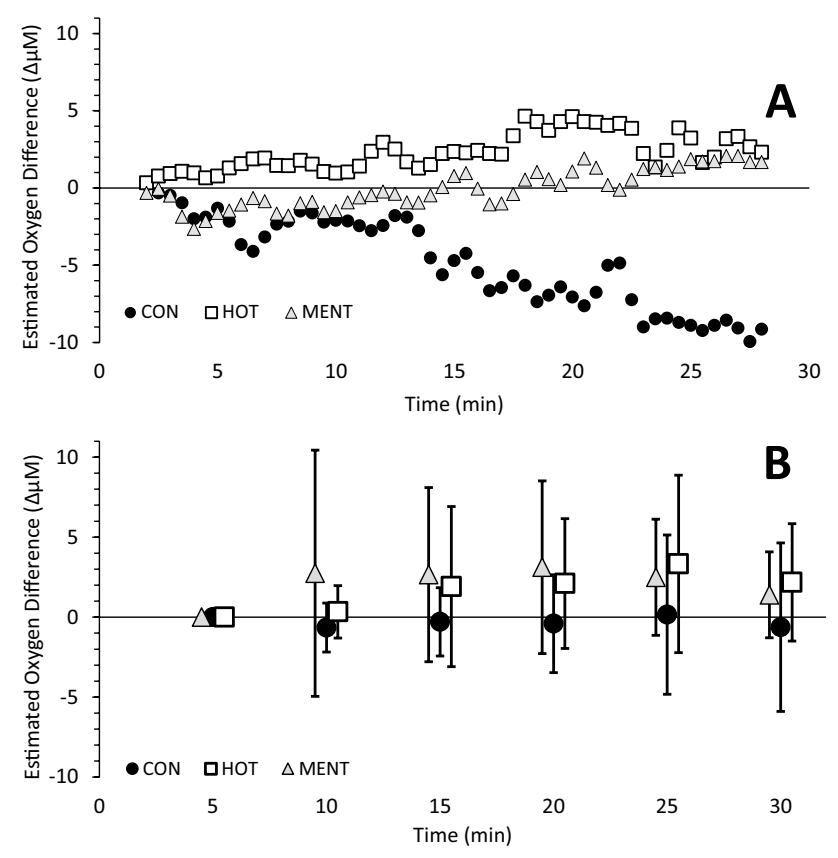

Fig. 4 Estimated oxygen difference in cerebral tissue, calculated using baseline-adjusted cerebral issue oxygenated and deoxygenated haemoglobin measured from the forehead using near-infrared spectroscopy. a displays an individual subjects data over time, while b displays the group means \pm SD. Readings were obtained while completing a battery of cognitive tasks during a 30-min heated walk while either normothermic (CON), hyperthermic (HOT) or hyperthermic with the aid of a menthol mouth-rinse (MENT). While there is an overall effect for CON to be lower than MENT and HOT (both $p<.025)$, no differences were found for analyses at each time point using the Holm p-value adjustment
- deoxygenated haemoglobin) increased $2.1 \pm 3.7 \mu \mathrm{M}$ and $2.6 \pm 4.1 \mu \mathrm{M}$ over the HOT and MENT trials compared to the CON trial $(\mathrm{CON}:-0.1 \pm 3.3 \mu \mathrm{M}$; HOT: $2.0 \pm 4.1 \mu \mathrm{M}$; MENT: $2.5 \pm 4.9 \mu \mathrm{M}$; CON vs. HOT: $p=0.025$; CON vs. MENT: $p=0.006$; HOT vs. MENT: $p=0.481$ ) (Fig. 4B), although no differences were evident for any individual time point (all $p>0.104)$.

\section{Theory of rising core temperature}

Due to methodological limitations providing the potential for participants to cool down during transition between the pre-heating facility and the heat chamber, there were occasions during the test where rectal temperature was declining. When tasks were re-analysed, excluding tasks completed while rectal temperature was declining, there remained no significance differences between conditions for any cognitive test (CON: $n=48$; MENT: $n=36$; HOT: $n=31$ ). Furthermore, when re-analysed using only participants whose rectal temperature was rising at a rate of $0.5^{\circ} \mathrm{C} . \mathrm{h}^{-1}$ or more, again no changes in significance were observed between conditions in the cognitive performance [CON: $n=47$ (8); MENT: $n=21$ (3); HOT: $n=21$ (5)].

\section{Discussion}

The primary aim of this study was to determine whether heat-related impairments in cognitive function were a product of core temperature, and second to determine whether a menthol mouth-rinse could mitigate any potential performance decline. The main findings indicate no measurable effect of moderately elevated core temperature on cognitive performance in a range of cognitive demands during exercise in the heat, therefore, there was also no demonstrable beneficial effect of menthol mouth-rinse as a cooling strategy.

To our knowledge, this is the first study to report the independent effects of core temperature on cognitive performance during exercise in a heated environment. Despite ensuring tests were conducted at a core temperature previously shown to impair elements of cognition, which induced greater physiological and heat-related perceptual strain in HOT and MENT compared to CON, no differences in cognitive performance were observed (Table 1, Fig. 3). The lack of relationship between core temperature and cognitive performance has previously been suggested (Taylor et al. 2016), but not tested. Therefore, it is likely that in field settings, other factors play a greater role in impairing cognition. Indeed, Hocking et al. (2001) used a similar experimental design, and found that preheating participants by having them walk in the heat $\left(40 \mathrm{~min}\right.$ at $5 \mathrm{~km} \cdot \mathrm{h}^{-1}$ in $35^{\circ} \mathrm{C}, 65 \%$ $\mathrm{RH})$ led to minimal changes in cognition. However, unlike the present study, Hocking et al. (2001) observed differences 
in working memory using the same digit span task. While impaired cognition was linked to higher core temperature in their study, the lack of such a finding in the current study (Table 1) indicates that fatigue or thermal discomfort from the exercise pre-heating method used by Hocking et al. (2001) may have played a larger role. Minimal changes were also observed by Caldwell et al. (2011) during cycling in the heat, between a group wearing military uniform compared to both a control group and a group wearing a cooling garment. The relatively low core temperatures reached $\left(\max \sim 38.3^{\circ} \mathrm{C}\right)$ may have limited the effects of heat on cognition, with it hypothesised that more stressful conditions might impair cognitive performance (Caldwell et al. 2011). However, in the current study, mean maximal core temperatures of $38.9^{\circ} \mathrm{C}$ also resulted in no effects on cognitive performance (Table 1), in line with another study that induced a similarly elevated core temperature using exercise in a hot environment (Caldwell et al. 2012). The core temperature at testing onset was based on when cognitive performance begins to decline (Schmit et al. 2017), with an expectation that core temperature would rise throughout the military march. Furthermore, the temperature at the start of exercise was chosen to prevent core temperature exceeding an ethical limit of $39.5^{\circ} \mathrm{C}$, as temperatures exceeding $40^{\circ} \mathrm{C}$ can risk heat stroke (Goforth and Kazman 2015).

While the selected threshold of $38.5^{\circ} \mathrm{C}$ may have been too low to compromise cognitive performance, it appears to have influenced cerebral activity (Fig. 4), as in both hyperthermic conditions, the estimated oxygenation difference increased. The increased cerebral oxygen requirement may have helped to preserve cognitive performance consistent with increases in cerebral oxygen extraction fraction previously seen during hyperthermic exercise (Rasmussen et al. 2010), alongside increased cerebral metabolic rate (González-Alonso et al. 2004, Rasmussen et al. 2010). Hocking et al. (2001) also showed alterations at the level of the brain to occur as a function of core temperature rather than ambient temperature. Using electroencephalography, it was shown for several tasks that there were transient increases in amplitude and decreases in latency when measuring steady-state visual evoked potentials in relevant areas of the brain (Hocking et al. 2001). Furthermore, studies using functional magnetic resonance imaging have shown executive function to be impaired under passive hyperthermia, and that brain activity is altered to support task performance, despite no changes occurring in the performance itself (Liu et al. 2013). Functional magnetic resonance imaging has also shown head cooling to mitigate negative effects of hyperthermia in some brain regions, including entire functional networks (Xue et al. 2018). In the current study, perceptual cooling used in MENT caused a reduction in deoxygenated haemoglobin, perhaps signifying reduced extraction (Fig. 4b). No concurrent perceptual differences were evident. Therefore, it remains unclear whether menthol may have any effect on cognitive performance, and cognitive activity, in a more stressful paradigm.

Although heat strain may have been insufficient to impair cognitive performance in the present study, an alternative theory suggests that the direction of change in core temperature is a more important factor in determining cognitive strain (Allan and Gibson 1979, Allan et al. 1979). Acknowledging this theory, we analysed only tasks completed while core temperature was rising and found no cognitive differences. One confounding factor that may have influenced this was that although rectal temperature was increasing in each condition, the rate of increase was far greater in CON (Fig. 2a). However, when only tasks that had a rate of rise in core temperature of $0.5^{\circ} \mathrm{C} . \mathrm{h}^{-1}$ or more were analysed, no differences in cognitive performance were observed. Whether the rate of rise in CON had the same effect as a higher absolute temperature in HOT and MENT cannot be discerned from the current data. To gain mechanistic insights as to how core temperature influences cognitive, and indeed physiological function, future studies should investigate the influence of the rate of rise in core temperature on cognition at different absolute temperatures.

\section{Conclusion}

In summary, no relationship was evident between core temperature and cognitive performance, which indicates that cognitive impairments reported during operations in such climates may be independent of moderately elevated core temperatures. Therefore, the use of heat mitigation strategies and devices are unlikely to improve cognition in hot environments. However, heat is known to accelerate the onset of fatigue and therefore how these factors interact with cognition likely requires further exploration. In the presence of thermal and exertional stresses, increases in the estimated oxygenation difference suggest an increased oxygen metabolism, which may be the underlying mechanism by which the brain adjusts to preserve cognitive performance.

Author contributions EA, JC, and AK conceptualised and designed the study. EA collected and analysed the data. EA wrote up the manuscript which was revised by JC and AK.

Funding The project was supported by a Vice-Chancellors Doctoral Scholarship obtained by EA.

Data availability Data and materials can be requested through the corresponding author.

Code availability Statistical code can be requested through the corresponding author. 


\section{Compliance with ethical standards}

\section{Conflict of interest N/A.}

Ethics approval Institutional ethical approval was obtained from the Auckland University of Technology Ethics Committee prior to participant recruitment (AUTEC 19/368).

Consent to participate All participants provided written consent to participate prior to any data collection.

Consent for publication All participants provided written consent for their data to be published.

\section{References}

Allan JR et al (1979a) Separation of the effects of raised skin and core temperature on performance of a pursuit rotor task. Aviat Space Environ Med 50:678-682

Allan JR et al (1979b) Effect of induced cyclic changes of deep body temperature on task performances. Aviat Space Environ Med 50:585-589

Arbuthnott K et al (2000) Trail Making Test, Part B as a Measure of Executive Control: Validation Using a Set-Switching Paradigm. J Clin Exp Neuropsychol 22:518-528

Bandelow S et al (2010) The effects of exercise, heat, cooling and rehydration strategies on cognitive function in football players. Scand J Med Sci Sports 20(Suppl 3):148-160

Bates D, et al. (2014). Fitting linear mixed-effects models using lme4. arXiv preprint arXiv: 1406.5823

Bedford T (1936) The warmth factor in comfort at work. London, Her Majesty's Stationery Office, a physiological study of heating and ventilation

Best R, et al. (2018) The development of a menthol solution for use during sport and exercise. Beverages 2

Bhattacharyya D et al (2017) Effect of load carriage and natural terrain conditions on cognitive performance in desert environments. Physiol Behav 179:253-261

Birrell SA et al (2010) The effect of load distribution within military load carriage systems on the kinetics of human gait. Appl Ergon 41(4):585-590

Boone J et al (2015) The impact of pedal rate on muscle oxygenation, muscle activation and whole-body $\mathrm{VO} 2$ during ramp exercise in healthy subjects. Eur J Appl Physiol 115(1):57-70

Borg GA (1982) Psychophysical bases of perceived exertion. Med Sci Sports Exerc 14(5):377-381

Caldwell JN et al (2011) The interaction of body armor, low-intensity exercise, and hot-humid conditions on physiological strain and cognitive function. Mil Med 176(5):488-493

Caldwell J et al (2012) Exertional thermal strain, protective clothing and auxiliary cooling in dry heat: evidence for physiological but not cognitive impairment. Eur J Appl Physiol 112(10):3597-3606

Cian C et al (2001) Effects of fluid ingestion on cognitive function after heat stress or exercise-induced dehydration. Int J Psychophysiol 42(3):243-251

Conklin HM et al (2018) Verbal working memory impairment in schizophrenia patients and their first-degree relatives: evidence from the digit span task. Am J Psychiatry 157(2):275-277

Cvirn MA et al (2019) The effects of hydration on cognitive performance during a simulated wildfire suppression shift in temperate and hot conditions. Appl Ergon 77:9-15
Eddy MD et al (2015) The effects of load carriage and physical fatigue on cognitive performance. PLoS ONE 10(7):e0130817

Flood TR et al (2017) Oral L-menthol reduces thermal sensation, increases work-rate and extends time to exhaustion, in the heat at a fixed rating of perceived exertion. Eur J Appl Physiol 117(7):1501-1512

Froom P et al (1993) Heat stress and helicopter pilot errors. J Occup Med 35(7):720-724

Ganio MS et al (2011) Mild dehydration impairs cognitive performance and mood of men. Br J Nutr 106(10):1535-1543

Gaoua N (2010) Cognitive function in hot environments: a question of methodology. Scand J Med Sci Sports 20(Suppl. 3):60-70

Gaoua N, et al. (2017). Perception, action, and cognition of football referees in extreme temperatures: impact on decision performance. Front Psychol 8: 1479

Gaoua N et al (2011) Alterations in cognitive performance during passive hyperthermia are task dependent. Int J Hyperthermia 27(1):1-9

Giambra L et al (2013) Performance on a sustained attention task as a function of strategy: a cross-sectional investigation using the Mackworth clock-test. Bull Psych Soc 26:333-335

Goforth CW et al (2015) Exertional heat stroke in navy and marine personnel: a hot topic. Crit Care Nurse 35(1):52-59

González-Alonso J et al (2004) Brain and central haemodynamics and oxygenation during maximal exercise in humans. J Physiol 557(1):331-342

Grego F et al (2005) Influence of exercise duration and hydration status on cognitive function during prolonged cycling exercise. Int $\mathrm{J}$ Sports Med 26(1):27-33

Hancock PA et al (2003) Effects of heat stress on cognitive performance: the current state of knowledge. Int J Hyperthermia 19(3):355-372

Hardy C et al (1989) "Not what, but how one feels: the measurement of affect during exercise". J Sport Exerc Psych 11:304-317

Head J et al (2017) Prior mental fatigue impairs marksmanship decision performance. Front Physiol 8:680

Hemmatjo R et al (2017) The effect of practical cooling strategies on physiological response and cognitive function during simulated firefighting tasks. Health Promot Perspect 7(2):66-73

Hocking $\mathrm{C}$ et al (2001) Evaluation of cognitive performance in the heat by functional brain imaging and psychometric testing. Comp Biochem Physiol A Mol Integr Physiol 128(4):719-734

Hoddes ED, Zarcone WV (1972) The development and use of the stanford sleepiness scale (SSS). Psychophysiology 9:150

Hunt AP et al (2016) Heat strain during military training activities: the dilemma of balancing force protection and operational capability. Temperature (Aust) 3:307-317

ISO (International Standards Organization) (1994) Moderate thermal environments-determination of the PMV and PPD indices and specification of the conditions forthermal comfort. International Standard ISO 7730, Geneva

Jiang QJ et al (2013) Hyperthermia impaired human visual short-term memory: an fMRI study. Int J Hyperth 29(3):219-224

Lee JKW et al (2014) Neck cooling and cognitive performance following exercise-induced hyperthermia. Eur J Appl Physiol 114(2):375-384

Lieberman HR et al (2005) Severe decrements in cognition function and mood induced by sleep loss, heat, dehydration, and undernutrition during simulated combat. Biol Psychiatry 57(4):422-429

Lieberman HR et al (2006) Cognition during sustained operations: comparison of a laboratory simulation to field studies. Aviat Space Environ Med 77(9):929-935

Liu K et al (2013) The impact of passive hyperthermia on human attention networks: an fMRI study. Behav Brain Res 15(243):220-230

Mackworth NH (1948) The breakdown of vigilance during prolonged visual search. Quart J Exp Psychol 1(1):6-21 
Martin K, et al. (2019) The impact of environmental stress on cognitive performance: a systematic review. Hum Factors 61:18720819839817

Martin K et al (2020) Physiological factors which influence cognitive performance in military personnel. Hum Factors 62(1):93-123

Mündel T et al (2010) The effects of swilling an L(-)-menthol solution during exercise in the heat. Eur J Appl Physiol 109(1):59-65

Navon D (1977) Forest before trees: The precedence of global features in visual perception. Cogn Psychol 9(3):353-383

Novak A (1996) Reaction time tests for science. https://appadvice.com/ app/reaction-time-tests-for-science/1148377237

Nolte HW et al (2013) Ad libitum vs. restricted fluid replacement on hydration and performance of military tasks. Aviat Space Environ Med 84(2):97-103

Owen G et al (2004) The role of blood glucose availability and fatigue in the development of cognitive impairment during combat training. Aviat Space Environ Med 75(3):240-246

Patterson $\mathrm{M}$ et al (1998) physical work and cognitive function during acute heat exposure before and after heat acclimation. Melbourne, Victoria, Defence Science and Technology Organisation

Piil JF et al (2017) Performance in complex motor tasks deteriorates in hyperthermic humans. Temperature (Aust) 4(4):420-428

Piil JF et al (2020) Direct exposure of the head to solar heat radiation impairs motor-cognitive performance. Scie Rep 10(1):7812

Posner MI et al (1984) Components of visual orienting. Attent Perform X Control Lang Process 32:531-556

Qian SW et al (2013) Altered topological patterns of large-scale brain functional networks during passive hyperthermia. Brain Cogn 83(1):121-131

Ramanathan N (1964) A new weighting system for mean surface temperature of the human body. J Appl Physiol 19(3):531-533

Rasmussen P et al (2010) Cerebral oxygenation is reduced during hyperthermic exercise in humans. Acta Physiol 199(1):63-70

Schmit $C$ et al (2017a) Cognitive functioning and heat strain: performance responses and protective strategies. Sports Med 47(7):1289-1302
Schmit C et al (2017b) Cognitive functioning and heat strain: performance responses and protective strategies. Sports Med 47(7):1289-1302

Stevens CJ et al (2016) Running performance and thermal sensation in the heat are improved with menthol mouth rinse but not ice slurry ingestion. Scand J Med Sci Sports 26(10):1209-1216

Strangman G et al (2006) Near-infrared spectroscopy and imaging for investigating stroke rehabilitation: test-retest reliability and review of the literature. Arch Phys Med Rehabil 87(12 Suppl 2):S12-19

Suzuki M et al (2004) Prefrontal and premotor cortices are involved in adapting walking and running speed on the treadmill: an optical imaging study. Neuroimage 23(3):1020-1026

Taylor L et al (2016) The impact of different environmental conditions on cognitive function: a focused review. Front Physiol 6:372-372

van Dokkum W et al (1996) Influence of a carbohydrate drink on performance of military personnel in NBC protective clothing. Aviat Space Environ Med 67(9):819-826

Vasmatzidis I et al (2002) An investigation of heat stress effects on time-sharing performance. Ergonomics 45(3):218-239

Vrijkotte $S$ et al (2016) Sustained military operations and cognitive performance. Aerosp Med Hum Perform 87(8):718-727

Williams D et al (1997) Effects of chemical protective clothing, exercise, and diphenhydramine on cognitive performance during sleep deprivation. Mil Psychol 9(4):329-358

Xue Y et al (2018) The effects of head-cooling on brain function during passive hyperthermia: an fMRI study. Int J Hyperthermia 34(7):1010-1019

Yanovich R et al (2015) Physiological and cognitive military related performances after 10-kilometer march. Disaster Mil Med 1:6-6

Publisher's Note Springer Nature remains neutral with regard to jurisdictional claims in published maps and institutional affiliations. 